\title{
A Six-Node Pentagonal Assumed Natural Strain Solid-Shell Element
}

\author{
K.Y.Sze , W.K.Chan \\ Department of Mechanical Engineering, The University of Hong Kong
}

Pokfulam Road, Hong Kong SAR, P.R.CHINA

\begin{abstract}
In this paper, a six-node pentagonal solid-shell element is formulated. Particular attention is focused on alleviating shear, trapezoidal and thickness lockings that plagues the conventional element. While assumed natural strain method is employed to alleviate shear and trapezoidal lockings, a modified generalized laminate stiffness matrix is proposed to circumvent thickness locking. Unlike the commonly adopted plane stress assumption, the modified laminate stiffness matrix enables the element to reproduce the exact thickness stress and transverse displacement when the element is loaded by thickness stress. Numerical examples reveal that the element is close in accuracy with other state-of-the-art three-node degenerated shell elements.
\end{abstract}

keywords : finite elements, solid shell, pentagonal, locking, laminate 


\section{INTRODUCTION}

Solid-Shell elements which possess no rotational d.o.f.s and are applicable to thin plate/shell analyses have attracted considerable attention [1-9]. Compared to the degenerated shell elements, solid-shell elements are advantageous in the following aspects. Firstly, solid-shell elements are simpler in their geometric and kinematic descriptions. Secondly, no special effort is required for matching the translations in solid elements and rotations in shell elements when a structure is composed of bulk and thin-walled regions. The laborious task of defining algebraic constraints or introducing solid-to-shell transition elements can be exempted. Thirdly, the complication on handling finite rotational increments in geometric nonlinear analyses can be avoided. Nevertheless, formulating robust solid-shell elements is indeed more demanding than formulating degenerated shell elements. While the latter elements are only bothered by shear and membrane lockings, the former elements are also plagued by trapezoidal and thickness lockings.

In previous solid-shell element formulations, shear and membrane lockings have been successively resolved by hybrid formulation [1,6], scaled hybrid formulation [2] and assumed natural strain method [4,5,7-9]. Trapezoidal locking occurs when lower order elements such as eight-node hexahedral elements are used to model curved shells so that their cross-sections assume the trapezoidal shape [10,11]. It can be resolved by scaled hybrid formulation [2] and assumed strain method $[4,5,8,9]$. On the other hand, thickness locking is caused by Poisson's ratio coupling of the inplane and transverse normal stress - normal strain responses. When the element is under pure bending load, the plane strain state instead of the physical plane stress state will be predicted. This locking phenomenon can be overcome by plane-stress enforcement in which the inplane and transverse normal stress - normal strain responses in the material compliance matrix are decoupled $[1,3,6,7]$, the enhanced assumed linear thickness strain modes [4,5,7] or hybrid-stress formulation $[2,8,12]$ with the assumed thickness stress independent of the transverse coordinate.

Most of the solid-shell elements possess two layers of nodes, the thickness strain derived from the nodal d.o.f.s can only be the thickness average of the physical thickness strain. Based on this property and the successfulness of hybrid-stress formulation in resolving thickness locking, a modified generalized laminate stiffness relation will be derived that can resolve thickness locking and some element abnormalities in laminate analyses. While a number of eight-node hexahedral solid-shell elements have been proposed [2,4,5,7-9], this paper derives a six-node pentagonal solidshell element which can work together with eight-node hexahedral solid-shell elements in meshes containing mixed element types [13-14]. Popular benchmark tests have been exercised. The computational results reveal that the proposed element is close in accuracy with other state-of-theart three-node degenerated plate/shell elements [15-17]. 


\section{THE CONVENTIONAL SIX-NODE SOLID-SHELL ELEMENT}

In this section, the formulation of the conventional six-node solid-shell element will be briefly described. With respect to nodal designation portrayed in Figure 1, the coordinate vector $\mathbf{X}$ and displacement vector $\mathbf{U}$ of the element are :

$$
\begin{aligned}
& \mathbf{X}(s, t, \zeta)=\mathbf{X}_{o}(s, t)+\zeta \mathbf{X}_{n}(s, t)=\sum_{i=1}^{3} N_{i} \cdot\left(\frac{1+\zeta}{2} \mathbf{X}_{i}+\frac{1-\zeta}{2} \mathbf{X}_{i+3}\right), \\
& \mathbf{U}(s, t, \zeta)=\mathbf{U}_{o}(s, t)+\zeta \mathbf{U}_{n}(s, t)=\sum_{i=1}^{3} N_{i} \cdot\left(\frac{1+\zeta}{2} \mathbf{U}_{i}+\frac{1-\zeta}{2} \mathbf{U}_{i+3}\right)
\end{aligned}
$$

where $N_{1}=r, N_{2}=s$ and $N_{3}=t ; r=1-s-t, s$ and $t$ are the area coordinates; $\zeta \in[-1,+1]$ is the transverse natural coordinate; $\mathbf{X}_{i}$ and $\mathbf{U}_{i}$ are the coordinate and displacement vectors of the i-th node. The infinitesimal natural strains with respect to $s, t$ and $\zeta$ can be derived as :

$$
\begin{aligned}
& \varepsilon_{s s}=\mathbf{X},_{s}^{T} \mathbf{U},_{s} \simeq \varepsilon_{s s}^{m}+\zeta \varepsilon_{s s}^{b}, \varepsilon_{t t}=\mathbf{X}_{{ }_{t}}^{T} \mathbf{U}_{{ }_{t}} \simeq \varepsilon_{t t}^{m}+\zeta \varepsilon_{t t}^{b}, \\
& 2 \varepsilon_{s t}=\mathbf{X}{ }_{s}^{T} \mathbf{U}_{{ }_{t}}+\mathbf{X}_{{ }_{t}}^{T} \mathbf{U},_{s} \simeq 2 \varepsilon_{s t}^{m}+2 \zeta \varepsilon_{s t}^{b}, \varepsilon_{\zeta \zeta}=\mathbf{X},_{\zeta}^{T} \mathbf{U}_{{ }_{\zeta}}=\mathbf{X}_{n}^{T} \mathbf{U}_{n}, \\
& \gamma_{\zeta s}=\mathbf{X},_{\zeta}^{T} \mathbf{U}_{,_{s}}+\mathbf{X}_{{ }_{s}}^{T} \mathbf{U}_{{ }_{\zeta}} \simeq \mathbf{X}_{n}^{T} \mathbf{U}_{o},_{s}+\mathbf{X}_{o}^{T},_{s} \mathbf{U}_{n}, \gamma_{\zeta t}=\mathbf{X}_{{ }_{\zeta}}^{T} \mathbf{U}_{{ }_{t}}+\mathbf{X}^{T},{ }_{{ }_{t}} \mathbf{U}_{{ }_{\zeta}}^{T} \simeq \mathbf{X}_{n}^{T} \mathbf{U}_{o}{ }_{t}+\mathbf{X}_{o}^{T}{ }_{t} \mathbf{U}_{n}
\end{aligned}
$$

in which " $m$ " and " $b$ " stand for membrane and bending, respectively. The approximations as indicated by " $\simeq$ " arise from the standard truncations of the second order $\zeta$-terms in the inplane strains $\left(\varepsilon_{s s}, \varepsilon_{t t}\right.$ and $\left.2 \varepsilon_{s t}\right)$ and the first order $\zeta$-terms in the transverse shear strains $\left(\gamma_{\zeta s}\right.$ and $\left.\gamma_{\zeta t}\right)$ in formulation of shell elements. It is trivial to show that the membrane and bending strains are constant inside the element.

Material properties are often specified with respect to a local physical coordinate system whose $\mathrm{x}-\mathrm{y}$ plane coincides with the element mid-plane defined by $\zeta=0$. To obtain the local physical strains from the natural strains, the following transformations are used :

$$
\begin{aligned}
& \boldsymbol{\epsilon}_{=}=\left\{\begin{array}{c}
\varepsilon_{x} \\
\varepsilon_{y} \\
\gamma_{x y}
\end{array}\right\}=\left[\begin{array}{rrc}
x,_{s}^{2} & x,_{t}^{2} & 2 x,{ }_{s} x,_{t} \\
y,_{s}^{2} & y,_{t}^{2} & 2 y,,_{s} y,_{t} \\
x, y,,_{s} & x,_{t} y,_{t} & x,,_{s} y,_{t}+x,_{t} y,_{s}
\end{array}\right]^{-T}\left\{\begin{array}{l}
\varepsilon_{s s} \\
\varepsilon_{t t} \\
\gamma_{s t}
\end{array}\right\}, \varepsilon_{z}=\varepsilon_{\|}=\frac{1}{z,{ }_{\zeta}^{2}} \varepsilon_{\zeta \zeta}, \\
& \boldsymbol{\gamma}=\left\{\begin{array}{l}
\gamma_{z x} \\
\gamma_{z y}
\end{array}\right\}=\left[\begin{array}{cc}
z,{ }_{\varsigma} x,,_{s} & z,{ }_{\varsigma} x,_{t} \\
z,,_{\varsigma} y,,_{s} & z,,_{\varsigma} y,_{t}
\end{array}\right]^{-T}\left\{\begin{array}{l}
\gamma_{\varsigma s} \\
\gamma_{\varsigma t}
\end{array}\right\}
\end{aligned}
$$


where $x,_{s}=\mathbf{e}_{x}^{T} \mathbf{X}_{o},_{s}, y,_{t}=\mathbf{e}_{y}^{T} \mathbf{X}_{o},_{t}, x,{ }_{t}=\mathbf{e}_{x}^{T} \mathbf{X}_{o},_{t}, y,_{s}=\mathbf{e}_{y}^{T} \mathbf{X}_{o},{ }_{s}$ and $z,{ }_{\zeta}=\mathbf{e}_{z}^{T} \mathbf{X}_{n} ; \mathbf{e}_{x}, \mathbf{e}_{y}$ and $\mathbf{e}_{z}$ are the unit vectors along the axis of the local Cartesian coordinates. By consolidating (2) and (3), the element strains can be expressed symbolically as :

$$
\boldsymbol{\epsilon}_{=}^{e}=\left(\mathbb{B}_{m}+\zeta \mathbb{B}_{b}\right) \mathbf{q}^{e}, \varepsilon_{\|}^{e}=\mathbb{B}_{\|} \mathbf{q}^{e}, \boldsymbol{\gamma}^{e}=\mathbb{B}_{t} \mathbf{q}^{e}
$$

where $\mathbf{q}^{e}=\left\{\mathbf{U}_{1}^{T}, \ldots, \mathbf{U}_{6}^{T}\right\}^{T}$ is the element displacement vector. The following uncoupled constitutive relations that cover a wide range of materials are assumed :

$$
\left\{\begin{array}{c}
\boldsymbol{\sigma}_{=} \\
\hdashline \sigma_{\|}
\end{array}\right\}=\left\{\begin{array}{c}
\sigma_{x} \\
\sigma_{y} \\
\sigma_{x y} \\
\hdashline \sigma_{z}
\end{array}\right\}=\left[\begin{array}{c:c}
\mathbf{C}_{=} & \mathbf{C}_{\times} \\
\hdashline \mathbf{C}_{\times}^{T} & \mathbf{C}_{\|}
\end{array}\right]\left\{\begin{array}{c}
\varepsilon_{x} \\
\varepsilon_{y} \\
2 \varepsilon_{x y} \\
\hdashline \varepsilon_{z}
\end{array}\right\}=\left[\begin{array}{c:c}
\mathbf{C}_{=} & \mathbf{C}_{\times} \\
\hdashline \mathbf{C}_{\times}^{T} & \mathbf{C}_{\|}
\end{array}\right]\left\{\begin{array}{c}
\boldsymbol{\epsilon}_{=} \\
\hdashline \varepsilon_{\|}
\end{array}\right\} \text {and } \boldsymbol{\tau}=\left\{\begin{array}{l}
\tau_{z x} \\
\tau_{z y}
\end{array}\right\}=\mathbf{C}_{t}\left\{\begin{array}{l}
\gamma_{z x} \\
\gamma_{z y}
\end{array}\right\}=\mathbf{C}_{t} \boldsymbol{\gamma}
$$

which simply require $\zeta$ or $z$ to be a principal material direction. By virtue of the minimum potential energy principle, (4) and (5), the element stiffness matrix can be derived as :

$$
\mathbb{K}^{e}=\int_{-1}^{+1} \int_{0}^{+1} \int_{0}^{1-t}\left(\left[\begin{array}{c}
\mathbb{B}_{m}+\zeta \mathbb{B}_{b} \\
\mathbb{B}_{\|}
\end{array}\right]^{T}\left[\begin{array}{c:c}
\mathbf{C}_{=} & \mathbf{C}_{\times} \\
\hdashline \mathbf{C}_{\times}^{T} & \mathbf{C}_{\|}
\end{array}\right]\left[\begin{array}{c}
\mathbb{B}_{m}+\zeta \mathbb{B}_{b} \\
\mathbb{B}_{\|}
\end{array}\right]+\mathbb{B}_{t}^{T} \mathbf{C}_{t} \mathbb{B}_{t}\right) J d s d t d \zeta
$$

In the above equation, $J$ is the Jacobian determinant for the global Cartesian and natural coordinate systems. It is usual that $J$ is approximated by $J_{o}=\left.J\right|_{\zeta=0}$. Moreover, it has been tested that the element prediction is insensitive to the approximation unless the mesh is extremely coarse with which the prediction is far from the converged value. After adopting $J \simeq J_{o}$,

$$
\mathbb{K}_{D I S P}^{e}=2 J_{o} \int_{0}^{+1} \int_{0}^{1-t}\left(\left[\begin{array}{l}
\mathbb{B}_{m} \\
\mathbb{B}_{\|} \\
\mathbb{B}_{b}
\end{array}\right]^{T} \mathbf{C}_{\perp}\left[\begin{array}{l}
\mathbb{B}_{m} \\
\mathbb{B}_{\|} \\
\mathbb{B}_{b}
\end{array}\right]+\mathbb{B}_{t}^{T} \mathbf{C}_{T} \mathbb{B}_{t}\right) d s d t
$$

where

$$
\mathbf{C}_{T}=\frac{1}{2} \int_{-1}^{+1} \mathbf{C}_{t} d \zeta \text { and } \mathbf{C}_{\perp}=\frac{1}{2} \int_{-1}^{+1}\left[\begin{array}{ccc}
\mathbf{C}_{=} & \mathbf{C}_{\times} & \zeta \mathbf{C}_{=} \\
\mathbf{C}_{\times}^{T} & C_{\|} & \zeta C_{\times}^{T} \\
\zeta \mathbf{C}_{=}^{T} & \zeta C_{\times} & \zeta^{2} \mathbf{C}_{=}
\end{array}\right] d \zeta \text { is the generalized laminate stiffness matrix }
$$

For the integrations with respect to the natural coordinates $s$ and $t$, the three-point integration rule is required.

\section{REMEDIES FOR SHEAR AND TRAPEZOIDAL LOCKINGS}

Shear locking is due to the excess number of transverse shear strains sampled in the process of integrating the element stiffness matrix. An effective method of resolving shear locking is the 
assumed natural strain (ANS) method in which the natural transverse shear strains are first sampled and then interpolated at some discrete element points $[15,18,19]$. Most of these sampled strains are common to the elements sharing the same edge. Thus, the number of independent shear strains in the system or global level can be reduced. For the element under consideration that possesses eighteen displacement modes, it contains six rigid body modes, three constant membrane strain modes and three constant bending strain modes. To stabilize the remaining six modes, at least three transverse shear strains and three thickness strains must be sampled. Similar to reference [15], the following natural transverse shear strains with respect to $r, s$ and $t$ are sampled at the mid-side points so as to ensure that the resulting element can pass the constant bending strain patch test (see Figure 2) :

$$
\begin{aligned}
& \bar{\gamma}_{\zeta r}=\left.\left(\mathbf{X}_{n}^{T}\left(\partial_{r} \mathbf{U}_{0}\right)+\left(\partial_{r} \mathbf{X}_{0}^{T}\right) \mathbf{U}_{n}\right)\right|_{t=r=1 / 2}, \bar{\gamma}_{\zeta s}=\left.\left(\mathbf{X}_{n}^{T}\left(\partial_{s} \mathbf{U}_{0}\right)+\left(\partial_{s} \mathbf{X}_{0}^{T}\right) \mathbf{U}_{n}\right)\right|_{r=s=1 / 2}, \\
& \bar{\gamma}_{\zeta t}=\left.\left(\mathbf{X}_{n}^{T}\left(\partial_{t} \mathbf{U}_{0}\right)+\left(\partial_{t} \mathbf{X}_{0}^{T}\right) \mathbf{U}_{n}\right)\right|_{s=t=1 / 2}
\end{aligned}
$$

in which the differentiation operators are defined as :

$$
\begin{aligned}
& \partial_{r} F(r, s, t)=\frac{\partial}{\partial r} F(r, s, 1-r-s), \partial_{s} F(r, s, t)=\frac{\partial}{\partial s} F(1-s-t, s, t), \\
& \partial_{t} F(r, s, t)=\frac{\partial}{\partial t} F(r, 1-r-t, t)
\end{aligned}
$$

By extrapolating the above strains to the element corners, the following physical transverse shear strains can be obtained :

$$
\begin{aligned}
& \tilde{\boldsymbol{\gamma}}_{I}=\left\{\begin{array}{l}
\tilde{\gamma}_{z x} \\
\tilde{\gamma}_{z y}
\end{array}\right\}_{I}=\frac{1}{\left.z_{\zeta}\right|_{s=t=0}}\left[\begin{array}{ll}
\partial_{r} x & \partial_{r} y \\
\partial_{s} x & \partial_{s} y
\end{array}\right]^{-1}\left\{\begin{array}{c}
\bar{\gamma}_{\zeta r} \\
\bar{\gamma}_{\zeta s}
\end{array}\right\} \quad \text { at the midpoint of nodes 1 and 4, } \\
& \tilde{\boldsymbol{\gamma}}_{I I}=\left\{\begin{array}{l}
\tilde{\gamma}_{z x} \\
\tilde{\gamma}_{z y}
\end{array}\right\}_{I I}=\frac{1}{\left.z_{\zeta}\right|_{t=r=0}}\left[\begin{array}{ll}
\partial_{s} x & \partial_{s} y \\
\partial_{t} x & \partial_{t} y
\end{array}\right]^{-1}\left\{\begin{array}{l}
\bar{\gamma}_{\zeta s} \\
\bar{\gamma}_{\zeta t}
\end{array}\right\} \quad \text { at the midpoint of nodes } 2 \text { and } 5, \\
& \tilde{\boldsymbol{\gamma}}_{I I}=\left\{\begin{array}{l}
\tilde{\gamma}_{z x} \\
\tilde{\gamma}_{z y}
\end{array}\right\}_{I I I}=\frac{1}{\left.z_{\zeta}\right|_{r=s=0}}\left[\begin{array}{ll}
\partial_{t} x & \partial_{t} y \\
\partial_{r} x & \partial_{r} y
\end{array}\right]^{-1}\left\{\begin{array}{c}
\bar{\gamma}_{\zeta t} \\
\bar{\gamma}_{\zeta r}
\end{array}\right\} \quad \text { at the midpoint of nodes } 3 \text { and } 6
\end{aligned}
$$

where $\partial_{r} x=\mathbf{e}_{x}^{T}\left(\partial_{r} \mathbf{X}_{0}\right), \partial_{r} y=\mathbf{e}_{y}^{T}\left(\partial_{r} \mathbf{X}_{0}\right), \partial_{s} x=\mathbf{e}_{x}^{T}\left(\partial_{s} \mathbf{X}_{0}\right), \partial_{s} y=\mathbf{e}_{y}^{T}\left(\partial_{s} \mathbf{X}_{0}\right), \partial_{t} x=\mathbf{e}_{x}^{T}\left(\partial_{t} \mathbf{X}_{0}\right)$ and $\partial_{t} y=\mathbf{e}_{y}^{T}\left(\partial_{t} \mathbf{X}_{0}\right)$ do not vary with the area coordinates. The interpolated physical transverse shear strain can be expressed as :

$$
\tilde{\gamma}=r \tilde{\gamma}_{I}+s \tilde{\gamma}_{I I}+t \tilde{\gamma}_{I I I}=\tilde{\mathbb{B}}_{t} \mathbf{q}^{e}
$$


where $\tilde{\mathbb{B}}_{t}$ is the modified transverse shear strain-displacement matrix.

Noteworthily, constraint index analysis presented by Saleeb et al [16] for three-node degenerated triangular facet-shell elements which directly [15] or indirectly [16,17] employ the natural transverse shear strains in (8) indicates that the elements still have a moderate chance of exhibiting shear locking. The solution suggested by Saleeb et al is to use macro-quadrilateral element formed by four triangular elements. The common nodes of the triangular elements is located such that one of the sampled natural transverse shear strains in the four triangular elements become dependent. Taking the quadrilateral I-II-III-IV in Figure 3 as an illustration, an arbitrary corner node, say node III, is first projected onto the plane defined by the remaining corner nodes. Node $\mathrm{V}$ which is the common nodes of the four triangular elements is taken to be the cross-diagonal points of the quadrilateral defined by I-II-III"-IV. By simple vector algebra, we have the following coordinate vectors for point III $^{*}$ and node $\mathrm{V}$ :

$$
\mathbf{X}_{I I I^{*}}=\mathbf{X}_{I I I}-\left(\left(\mathbf{X}_{I I I}-\mathbf{X}_{I}\right)^{T} \mathbf{n}\right) \mathbf{n} \quad \text { and } \quad \mathbf{X}_{V}=\mathbf{X}_{I}+\frac{\left(\mathbf{X}_{I I}-\mathbf{X}_{I}\right)^{T} \mathbf{f}}{\left(\mathbf{X}_{I I I^{*}}-\mathbf{X}_{I}\right)^{T} \mathbf{f}}\left(\mathbf{X}_{I I I^{*}}-\mathbf{X}_{I}\right)
$$

where

$$
\mathbf{n}=\frac{\left(\mathbf{X}_{I I}-\mathbf{X}_{I}\right) \times\left(\mathbf{X}_{I V}-\mathbf{X}_{I}\right)}{\left\|\left(\mathbf{X}_{I I}-\mathbf{X}_{I}\right) \times\left(\mathbf{X}_{I V}-\mathbf{X}_{I}\right)\right\|} \quad \text { and } \quad \mathbf{f}=\left(\mathbf{X}_{I V}-\mathbf{X}_{I I}\right) \times \mathbf{n}
$$

The same scheme can be equally applied to the present six-node triangular prismatic element for enhancing the element immunity with respect to shear locking, namely, a macro-hexahedral element formed by four triangular prisms. Interior nodes on the top and bottom quadrilateral faces of the macro-element are defined by the afore-mentioned scheme.

Similarly to shear locking, the excessive number of sampled thickness strains which lead to trapezoidal locking $[8,11]$ can be reduced in the system level by sampling the strain along the element edges $[4,5,8,9]$, namely

$$
\tilde{\boldsymbol{\varepsilon}}_{\|}=\left.r \tilde{\boldsymbol{\varepsilon}}_{\|}\right|_{s=t=0}+\left.s \tilde{\boldsymbol{\varepsilon}}_{\|}\right|_{t=r=0}+\left.t \tilde{\boldsymbol{\varepsilon}}_{\|}\right|_{r=s=0}=\tilde{\mathbb{B}}_{\|} \mathbf{q}^{e}
$$

where $\tilde{\mathbb{B}}_{\|}$is the modified thickness strain-displacement matrix. With $\tilde{\mathbb{B}}_{t}$ and $\tilde{\mathbb{B}}_{\|}$, the modified element stiffness matrices would be :

$$
\mathbb{K}_{A N S}^{e}=2 J_{o} \int_{0}^{+1} \int_{0}^{1-t}\left(\left[\begin{array}{l}
\mathbb{B}_{m} \\
\tilde{\mathbb{B}}_{\|} \\
\mathbb{B}_{b}
\end{array}\right]^{T} \mathbf{C}_{\perp}\left[\begin{array}{l}
\mathbb{B}_{m} \\
\tilde{B}_{\|} \\
\mathbb{B}_{b}
\end{array}\right]+\tilde{\mathbb{B}}_{t}^{T} \mathbf{C}_{T} \tilde{\mathbb{B}}_{t}\right) d s d t
$$

The above element is free from trapezoidal locking and possesses the same immunity to shear 
locking as the other state-of-the-art three-node degenerated shell elements [15-17].

\section{REMEDY FOR THICKNESS LOCKING}

When an element is subjected to inplane pure bending, the bending strain is a linear function of the transverse coordinate $\zeta$. Poisson's ratio coupling requires the thickness strain to be a linear function of $\zeta$ in order that plane stress condition can be assumed. Most, if not all, solid-shell elements have only two layers of nodes and thus the thickness strain does not vary with $\zeta$. Without any treatment, these elements fail in reproducing the expected plane stress condition. Successful treatments includes the enforcement of the plane stress condition by decoupling the thickness stress- thickness strain response in the material compliance matrix $[1,3,6,7]$ and enhancing the thickness strain with linear terms [4,5,7]. Moreover, the hybrid-stress formulation with the assumed thickness stress independent of $\zeta$ is also an effective remedy $[2,8,12]$. For the assumed stress, it can only assume the thickness average of the displacement-derived stress. Apparently, the average is zero under pure inplane bending and the plane stress condition can be reproduced. Among the afore-mentioned treatments, the most convenient one is the enforcement of plane stress condition which unfortunately induces discontinuity of the thickness stress when the element is sandwiched by conventional solid elements, see Figure 4. Being inspired by the success of the hybrid-stress formulation, a new method to overcome thickness locking is here proposed.

It can be noted that the two generalized material stiffness matrices in (7) define the following generalized element stresses :

$$
\left\{\begin{array}{c}
\overline{\boldsymbol{\sigma}}_{m}^{e} \\
\bar{\sigma}_{\|}^{e} \\
\overline{\boldsymbol{\sigma}}_{b}^{e}
\end{array}\right\}=\frac{1}{2} \int_{-1}^{+1}\left\{\begin{array}{c}
\boldsymbol{\sigma}_{=}^{e} \\
\sigma_{\|}^{e} \\
\zeta \boldsymbol{\sigma}_{=}^{e}
\end{array}\right\} d \zeta=\frac{1}{2} \int_{-1}^{+1}\left\{\begin{array}{c}
\mathbf{C}_{=} \boldsymbol{\epsilon}_{=}^{e}+\mathbf{C}_{x} \varepsilon_{\|}^{e} \\
\mathbf{C}_{x}^{T} \boldsymbol{\epsilon}_{=}^{e}+\mathbf{C}_{\|} \varepsilon_{\|}^{e} \\
\zeta\left(\mathbf{C}_{=} \boldsymbol{\epsilon}_{=}^{e}+\mathbf{C}_{x} \varepsilon_{\|}^{e}\right)
\end{array}\right\} d \zeta=\mathbf{C}_{\perp}\left\{\begin{array}{c}
\boldsymbol{\epsilon}_{m}^{e} \\
\varepsilon_{\|}^{e} \\
\boldsymbol{\epsilon}_{b}^{e}
\end{array}\right\} \quad, \quad \overline{\boldsymbol{\tau}}=\frac{1}{2} \int_{-1}^{+1} \mathbf{C}_{t} d \zeta \cdot \boldsymbol{\gamma}^{e}=\mathbf{C}_{T} \cdot \boldsymbol{\gamma}^{e}
$$

Realizing that the advent of thickness locking is induced by the constant nature of $\varepsilon_{\|}^{e}$ with respect to $\zeta$, the constitutive relation in (5) is first rewritten as :

$$
\left\{\begin{array}{c}
\boldsymbol{\sigma}_{=} \\
\hdashline \varepsilon_{\|}
\end{array}\right\}=\left[\begin{array}{c:c}
\mathbf{A} & \mathbf{B} \\
\hdashline-\mathbf{B}^{T} & \mathbf{D}
\end{array}\right]\left\{\begin{array}{c}
\boldsymbol{\epsilon}_{=} \\
\hdashline \sigma_{\|}
\end{array}\right\}
$$

As the element thickness strain $\varepsilon_{\|}^{e}$ can only represent the thickness average of the pointwise thickness strain that derived from the element inplane strain and thickness stress, we have 


$$
\varepsilon_{\|}^{e}=\frac{1}{2} \int_{-1}^{+1}\left(\left[\begin{array}{ll}
-\mathbf{B}^{T} & D
\end{array}\right]\left\{\frac{\boldsymbol{\epsilon}_{m}^{e}+\zeta \boldsymbol{\epsilon}_{b}^{e}}{\sigma_{\|}^{e}}\right\}\right) d \zeta
$$

Same as the hybrid-stress method, the element thickness stress is here taken to be independent of $\zeta$. The assumption not only resolves the thickness locking but also improves the element response when laminated materials are modelled as the traction continuity along $\zeta$ inside the element is ensured. With the assumption,

$$
\begin{aligned}
& \varepsilon_{\|}^{e}=\left[\begin{array}{lll}
-\mathbf{B}_{0}^{T} & \mathbf{D}_{0} & -\mathbf{B}_{1}^{T}
\end{array}\right]\left\{\begin{array}{c}
\boldsymbol{\epsilon}_{m}^{e} \\
\sigma_{\|}^{e} \\
\boldsymbol{\epsilon}_{b}^{e}
\end{array}\right\}, \\
& \left.\left\{\begin{array}{c}
\overline{\boldsymbol{\sigma}}_{m}^{e} \\
\overline{\boldsymbol{\sigma}}_{b}^{e}
\end{array}\right\}=\frac{1}{2} \int_{-1}^{+1}\left\{\begin{array}{c}
\boldsymbol{\sigma}_{=}^{e} \\
\zeta \boldsymbol{\sigma}_{=}^{e}
\end{array}\right\} \mathrm{d} \zeta=\frac{1}{2} \int_{-1}^{+1}\left[\begin{array}{cc}
\mathbf{A} & \mathbf{B} \\
\zeta \mathbf{A} & \zeta \mathbf{B}
\end{array}\right]\left\{\begin{array}{c}
\boldsymbol{\epsilon}_{m}^{e}+\zeta \boldsymbol{\epsilon}_{b}^{e} \\
\hdashline \sigma_{\|}^{e}
\end{array}\right\}\right) \mathrm{d} \zeta=\left[\begin{array}{lll}
\mathbf{A}_{0} & \mathbf{B}_{0} & \mathbf{A}_{1} \\
\mathbf{A}_{1} & \mathbf{B}_{1} & \mathbf{A}_{2}
\end{array}\right]\left\{\begin{array}{l}
\boldsymbol{\epsilon}_{m}^{e} \\
\sigma_{\|}^{e} \\
\boldsymbol{\epsilon}_{b}^{e}
\end{array}\right\}
\end{aligned}
$$

where $\left[\mathbf{A}_{0}, \mathbf{A}_{1}, \mathbf{A}_{2}, \mathbf{B}_{0}, \mathbf{B}_{1}, \mathbf{D}_{0}\right]=\frac{1}{2} \int_{-1}^{+1}\left[\mathbf{A}, \zeta \mathbf{A}, \zeta^{2} \mathbf{A}, \mathbf{B}, \zeta \mathbf{B}, \mathbf{D}\right] \mathrm{d} \zeta$. A modified generalized laminate stiffness matrix $\tilde{\mathbf{C}}_{\perp}$ is derived by changing the equation objects of (17) as :

$$
\tilde{\mathbf{C}}_{\perp}=\left[\begin{array}{ccc}
\mathbf{A}_{0}+\mathbf{B}_{0} \mathbf{D}_{0}^{-1} \mathbf{B}_{0}^{T} & \mathbf{B}_{0} \mathbf{D}_{0}^{-1} & \mathbf{A}_{1}+\mathbf{B}_{0} \mathbf{D}_{0}^{-1} \mathbf{B}_{1}^{T} \\
\mathbf{D}_{0}^{-1} \mathbf{B}_{0}^{T} & \mathbf{D}_{0}^{-1} & \mathbf{D}_{0}^{-1} \mathbf{B}_{1}^{T} \\
\mathbf{A}_{1}+\mathbf{B}_{1} \mathbf{D}_{0}^{-1} \mathbf{B}_{0}^{T} & \mathbf{B}_{1} \mathbf{D}_{0}^{-1} & \mathbf{A}_{2}+\mathbf{B}_{1} \mathbf{D}_{0}^{-1} \mathbf{B}_{1}^{T}
\end{array}\right] \text { and }\left\{\begin{array}{c}
\overline{\boldsymbol{\sigma}}_{m}^{e} \\
\sigma_{\|}^{e} \\
\overline{\boldsymbol{\sigma}}_{b}^{e}
\end{array}\right\}=\tilde{\mathbf{C}}_{\perp}\left\{\begin{array}{c}
\boldsymbol{\epsilon}_{m}^{e} \\
\varepsilon_{\|}^{e} \\
\boldsymbol{\epsilon}_{b}^{e}
\end{array}\right\}
$$

Using (18), the element stiffness matrix in (13) becomes :

$$
\mathbb{\mathbb { K } _ { A N S _ { - } m C } ^ { e }}=2 J_{o} \int_{0}^{+1} \int_{0}^{1-t}\left(\left[\begin{array}{c}
\mathbb{B}_{m} \\
\tilde{\mathbb{B}}_{\|} \\
\mathbb{B}_{b}
\end{array}\right]^{T} \tilde{\mathbf{C}}_{\perp}\left[\begin{array}{c}
\mathbb{B}_{m} \\
\tilde{\mathbb{B}}_{\|} \\
\mathbb{B}_{b}
\end{array}\right]+\tilde{\mathbb{B}}_{t}^{T} \mathbf{C}_{T} \tilde{\mathbb{B}}_{t}\right) d s d t
$$

While it is straight forward to determine the transverse shear stress in the post-processing stage, (18) is first employed to calculate $\sigma_{\|}^{e}$ which together with the pointwise inplane strain are then used in (15) for computing the pointwise inplane stress.

\section{NUMERICAL BENCHMARK TESTS}

In the section, a number of popular benchmark tests for plate and shell element models will be exercised. Results of the following elements will also be included for comparison :

- DISP_PS - same as $\mathbb{K}_{D I S P}^{e}$ in (7) except that the plane stress enforcement is employed.

- $\quad$ ANS - given in (13). 
- ANS_PS - same as ANS except that the plane stress enforcement is employed.

- ANS_PS* - same as ANS_PS except that the truncated second order $\zeta$-terms in the inplane strain and the truncated $\zeta$-terms in the Jacobian determinant are included in the formulation. Second order Gaussian quadrature is employed for the thickness integration. For flat plate problems, ANS_PS and ANS_PS* are identical.

- ANS_mC - given in (19).

- HMSH3 - a state-of-the-art three-node hybrid-stress degenerated shell element proposed by Saleeb et al [16].

All elements are integrated by the three-point rule and thus are rank sufficient. Unless specified otherwise, the predictions are normalized by the reference solutions provided by MacNeal \& Harder [20] and the mesh density employed to model the subsequent problems will be quantified by the number of cross-diagonal hexahedral macroelements, see (11) and Figure 3.

Patch Tests - MacNeal \& Harder's patch tests for degenerated shell elements are exercised and the results are listed in Table 1 [20]. All the elements pass the constant membrane strain patch test. As constant modes are not admissible in its assumed transverse shear stress field, HMSH3 fails the constant transverse shear strain patch test. Without using the assumed natural strain formulation, DISP_PS fails the constant bending strain patch test. Poisson's ratio coupling effect leads to thickness locking in ANS and, thus, fails the element in the constant bending strain patch test for non-zero Poisson's ratio. Only ANS_PS, ANS_PS* and ANS_mC pass all the patch tests. 
Table 1. Results of patch tests.

\begin{tabular}{|c|c|c|c|c|}
\hline Element Model & Membrane & Transverse Shear & Bending, $v=0$ & Bending, $v \neq 0$ \\
\hline DISP_PS & Pass & Pass & Fail & Fail \\
\hline ANS & Pass & Pass & Pass & Fail \\
\hline ANS_PS & Pass & Pass & Pass & Pass \\
\hline ANS_mC & Pass & Pass & Pass & Pass \\
\hline HMSH3 & Pass & Fail & Pass & Pass \\
\hline
\end{tabular}

\# ANS_PS and ANS_PS* are identical in this problem.

Responses to Compressive Loading - A right angle isosceles triangular prism of dimension $10 \times 10 \times 2$ is considered as shown in Figure 5. The prism is composed of two plies of isotropic materials and is modelled by one element. It rests on a rigid floor and all nodes are restrained from horizontal movement. Compressive forces equivalent to 0.6 unit of compressive stress are applied to the upper element nodes. Tables $2 \mathrm{a}$ and $2 \mathrm{~b}$ list the vertical deflection of the upper element nodes and the three normal stresses which are uniform inside each of the two plies for two material configurations. Subscripts " $T$ " and " $B$ " refer to the material properties in the top and bottom plies, respectively. Only ANS_mC can yield the exact solutions.

Table 2a. Element predictions for compressive loading with $E^{T}=E^{B}=10$ and $v^{T}=v^{B}=0.3$.

\begin{tabular}{|c|c|c|c|c|}
\hline & deflection & $\sigma_{X}^{T}\left(=\sigma_{X}^{B}\right)$ & $\sigma_{Y}^{T}\left(=\sigma_{Y}^{B}\right)$ & $\sigma_{Z}^{T}\left(=\sigma_{Z}^{B}\right)$ \\
\hline DISP_PS & 0.12 & 0 & 0 & 0.6 \\
\hline ANS & 0.08914 & 0.2571 & 0.2571 & 0.6 \\
\hline ANS_mC & 0.08914 & 0.2571 & 0.2571 & 0.6 \\
\hline & 0.08914 & 0.2571 & 0.2571 & 0.6 \\
exact & & & & \\
\hline
\end{tabular}

\# predictions of DISP_PS, ANS_PS and ANS_PS* are identical in this problem.

Table 2b. Element predictions for compressive loading with $E^{T}=10, E^{B}=1, v^{T}=0.3$ and $v^{B}=0.2$.

\begin{tabular}{|c|c|c|c|c|c|c|c|}
\hline & $\begin{array}{c}\text { deflectio } \\
\mathrm{n}\end{array}$ & $\sigma_{X}^{T}$ & $\sigma_{Y}^{T}$ & $\sigma_{Z}^{T}$ & $\sigma_{X}^{B}$ & $\sigma_{Y}^{B}$ & $\sigma_{Z}^{B}$ \\
\hline DISP_PS & 0.2182 & 0 & 0 & 0.4196 & 0 & 0 & 0.04545 \\
\hline ANS & 0.1647 & 0.4751 & 0.4751 & 1.1085 & 0.02287 & 0.02287 & 0.09150 \\
\hline ANS_mC & 0.5846 & 0.2571 & 0.2571 & 0.6 & 0.15 & 0.15 & 0.6 \\
\hline exact & 0.5846 & 0.2571 & 0.2571 & 0.6 & 0.15 & 0.15 & 0.6 \\
\hline
\end{tabular}

\# predictions of DISP_PS, ANS_PS and ANS_PS* are identical in this problem. 
Square Plate Problems - Simply-supported and clamped square plates of side length $L$ and thickness $h$ are considered. Owing to symmetry, only a quarter of the plate is modelled as shown in Figure 6. The element predictions after normalized by the thin plate solutions [21] are given in Tables $3 \mathrm{a}$ and 3b. Besides DISP_PS, all other elements are insensitive to the aspect ratio. Though ANS does not suffer from shear locking, its accuracy is inferior to that of ANS_PS, ANS_mC and HMSH3.

Table 3a. Normalized central deflections for simply-supported square plate under uniform load.

\begin{tabular}{|c|c|c|c|c|c|c|}
\hline$L / h$ & Mesh & DISP_PS & ANS & ANS_PS & ANS_mC & HMSH3 \\
\hline \multirow{3}{*}{$10^{2}$} & $2 \times 2$ & 0.010 & 0.746 & 0.913 & 0.913 & 0.913 \\
& $4 \times 4$ & 0.042 & 0.800 & 0.980 & 0.980 & 0.980 \\
& $8 \times 8$ & 0.145 & 0.814 & 0.997 & 0.997 & 0.995 \\
\hline \multirow{3}{*}{$10^{3}$} & $2 \times 2$ & 0.000 & 0.745 & 0.912 & 0.912 & 0.912 \\
& $4 \times 4$ & 0.000 & 0.798 & 0.978 & 0.978 & 0.978 \\
& $8 \times 8$ & 0.002 & 0.812 & 0.995 & 0.995 & 0.994 \\
\hline \multirow{3}{*}{$10^{4}$} & $2 \times 2$ & 0.000 & 0.745 & 0.912 & 0.912 & 0.912 \\
& $4 \times 4$ & 0.000 & 0.798 & 0.978 & 0.978 & 0.978 \\
& $8 \times 8$ & 0.000 & 0.812 & 0.995 & 0.995 & 0.994 \\
\hline
\end{tabular}

\# ANS_PS and ANS_PS* are identical in this problem.

Table 3b. Normalized central deflections for clamped square plate under uniform load.

\begin{tabular}{|c|c|c|c|c|c|c|}
\hline$L / h$ & Mesh & DISP_PS & ANS & ANS_PS & ANS_mC & HMSH3 \\
\hline \multirow{3}{*}{$10^{2}$} & $2 \times 2$ & 0.010 & 0.638 & 0.781 & 0.781 & 0.781 \\
& $4 \times 4$ & 0.034 & 0.773 & 0.946 & 0.946 & 0.947 \\
& $8 \times 8$ & 0.117 & 0.809 & 0.991 & 0.991 & 0.991 \\
\hline \multirow{3}{*}{$10^{3}$} & $2 \times 2$ & 0.000 & 0.635 & 0.778 & 0.778 & 0.775 \\
& $4 \times 4$ & 0.000 & 0.770 & 0.943 & 0.943 & 0.939 \\
& $8 \times 8$ & 0.001 & 0.806 & 0.987 & 0.987 & 0.987 \\
\hline \multirow{3}{*}{$10^{4}$} & $2 \times 2$ & 0.000 & 0.635 & 0.778 & 0.778 & 0.775 \\
& $4 \times 4$ & 0.000 & 0.770 & 0.943 & 0.943 & 0.939 \\
& $8 \times 8$ & 0.000 & 0.806 & 0.987 & 0.987 & 0.987 \\
\hline
\end{tabular}

\# ANS_PS and ANS_PS* are identical in this problem.

Circular Plate Problems - Meshes for modelling a quadrant of a circular plate is shown in Figure 7. The numbers of hexahedral macroelements $N$ 's employed include 3,12 and 48. Two radius to 
thickness ratio $(R / h)$ are examined. Table $4 \mathrm{a}$ and $4 \mathrm{~b}$ shows the normalized deflections with respect to the thin plate solutions [21] for different $R$ to $h$ ratios, boundary and loading conditions. The relative performance of the elements is similar to that in the square plate problem.

Table 4a: Normalized central deflections for circular plate under point load.

\begin{tabular}{|c|c|c|c|c|c|c|c|}
\hline & $R / h$ & $N$ & DISP_PS & ANS & ANS_PS & ANS_mC & HMSH3 \\
\hline \multirow{3}{*}{$\begin{array}{c}\text { simply- } \\
\text { supported }\end{array}$} & \multirow{3}{*}{100} & 3 & 0.011 & 0.693 & 0.910 & 0.910 & 0.914 \\
& & 12 & 0.042 & 0.747 & 0.971 & 0.971 & 0.971 \\
& \multirow{2}{*}{1000} & 48 & 0.147 & 0.766 & 0.993 & 0.992 & 0.994 \\
& & 3 & 0.000 & 0.704 & 0.905 & 0.913 & 0.911 \\
& & 48 & 0.000 & 0.732 & 0.962 & 0.973 & 0.968 \\
& \multirow{3}{*}{100} & 3 & 0.000 & 0.773 & 0.986 & 0.982 & 0.990 \\
\cline { 2 - 7 } clamped & 12 & 0.032 & 0.723 & 0.885 & 0.882 & 0.886 \\
& & 48 & 0.111 & 0.794 & 0.971 & 0.969 & 0.972 \\
\cline { 2 - 7 } & \multirow{3}{*}{1000} & 3 & 0.000 & 0.485 & 0.593 & 0.594 & 0.593 \\
& & 12 & 0.000 & 0.715 & 0.877 & 0.880 & 0.878 \\
& & 48 & 0.000 & 0.789 & 0.964 & 0.967 & 0.964 \\
\hline
\end{tabular}

\# ANS_PS and ANS_PS* are identical in this problem.

Table 4b: Normalized central deflections for circular plate under uniform load.

\begin{tabular}{|c|c|c|c|c|c|c|c|}
\hline & $R / h$ & $N$ & DISP_PS & ANS & ANS_PS & ANS_mC & HMSH3 \\
\hline \multirow{6}{*}{$\begin{array}{l}\text { simply- } \\
\text { supported }\end{array}$} & \multirow{3}{*}{100} & 3 & 0.010 & 0.694 & 0.917 & 0.917 & 0.918 \\
\hline & & 12 & 0.042 & 0.746 & 0.981 & 0.981 & 0.982 \\
\hline & & 48 & 0.152 & 0.757 & 0.996 & 0.996 & 0.996 \\
\hline & \multirow{3}{*}{1000} & 3 & 0.000 & 0.703 & 0.940 & 0.913 & 0.917 \\
\hline & & 12 & 0.000 & 0.751 & 0.962 & 0.985 & 0.981 \\
\hline & & 48 & 0.000 & 0.751 & 0.995 & 0.994 & 0.995 \\
\hline \multirow{6}{*}{ clamped } & \multirow{3}{*}{100} & 3 & 0.008 & 0.578 & 0.708 & 0.708 & 0.709 \\
\hline & & 12 & 0.028 & 0.756 & 0.925 & 0.925 & 0.926 \\
\hline & & 48 & 0.103 & 0.802 & 0.982 & 0.982 & 0.983 \\
\hline & \multirow{3}{*}{1000} & 3 & 0.000 & 0.577 & 0.708 & 0.704 & 0.706 \\
\hline & & 12 & 0.000 & 0.751 & 0.920 & 0.918 & 0.924 \\
\hline & & 48 & 0.000 & 0.800 & 0.978 & 0.977 & 0.980 \\
\hline
\end{tabular}

\# ANS_PS and ANS_PS* are identical in this problem. 
Homogeneous and Laminated Ring-Shell Pinched by Line Forces - This problem is depicted in Figure 8. Six quadrilateral macroelements are employed to model one quarter of the ring-shell. Two isotropic materials " $A$ " and " $B$ " with properties $E_{A}=10, v_{A}=0.3, E_{B}=1$ and $v_{B}=0$ are considered. The homogeneous ring is made of material $A$. Two-ply and three-ply laminations A/B and A/B/A are also considered. In each lamination, all plies are of equal thickness. Two radius $(R)$ to thickness (h) ratios are examined. The vertical deflections under the line force are computed, normalized by the thin curved beam solutions and listed in Table 5.

Table 5: Normalized deflection under line force for pinched ring-shell

\begin{tabular}{|c|c|c|c|c|c|}
\hline Lamination & $R / h$ & DISP_PS & ANS & ANS_PS & ANS_mC \\
\hline A & 20 & 0.091 & 0.887 & 0.981 & 0.981 \\
& 100 & 0.004 & 0.883 & 0.976 & 0.976 \\
\hline \multirow{2}{*}{$A / B$} & 20 & 0.049 & 0.933 & 1.007 & 1.003 \\
& 100 & 0.002 & 0.909 & 0.981 & 0.980 \\
\hline$A / B / A$ & 20 & 0.121 & 0.890 & 0.983 & 0.984 \\
& 100 & 0.005 & 0.884 & 0.977 & 0.977 \\
\hline
\end{tabular}

Pinched Cylinder - This problem is depicted in Figure 9. The cylinder is mounted on two rigid end diaphragms and loaded by a pair of diametrically opposite point loads. One-eighth of the structure is analyzed and meshed by $N \times N$ quadraliteral macroelements. Displacement components in the $\mathrm{x}$ and $z$ - directions are restrained for the lower layers of nodes over the diaphragms. The normalized deflections of point A are plotted. Predictions of ANS_PS, ANS_PS* and ANS_mC are graphically indistinguishable and thus are represented by one line. Their accuracy is very close to that of HMSH3.

Scordelis-Lo Cylinder Roof - This problem is depicted in Figure 10. The roof is supported on two rigid end diaphragms and loaded by its own weight. Owing to symmetry, only a quarter of the roof is modelled. The normalized deflections of point A are shown. With Poisson's ratio equal to zero, ANS, ANS_PS and ANS_mC are identical. Moreover, results of ANS_PS* are graphically indistinguishable from that of ANS_PS and better than that of HMSH3.

Hemispherical Shell - Figure 11 shows a hemispherical shell with a $18^{\circ}$ cutout. The equator is subjected to anti-symmetric point loads along the coordinate axes. The normalized radial deflections at point A are plotted. Predictions of ANS_PS, ANS_PS* and ANS_mC are graphically 
indistinguishable and are better than that of HMSH3.

Cantilever Panel subjected to Inplane End Shear Forces - Figure 12 shows a $4 \times 1 \times h$ cantilever panel. Inplane shear force is applied to the free end. Using the plane elasticity solution [22] as the reference, Table 6 lists the normalized end deflections for different thickness and Poisson's ratios. Same as the constant strain triangle, none of the elements perform well in this example. Since the dilation constraint can be accommodated by varying the thickness strain, the elements do not exhibit dilatational locking in this problem.

Table 6 : Normalized end deflections for cantilever panel subjected to inplane end shear force

\begin{tabular}{|c|c|c|c|c|c|c|}
\hline \multirow{2}{*}{} & \multicolumn{3}{|c|}{$h=0.2$} & \multicolumn{3}{c|}{$h=0.02$} \\
\cline { 2 - 7 } & $v=0.3$ & $v=0.49$ & $v=0.4999$ & $v=0.3$ & $v=0.49$ & $v=0.4999$ \\
\hline DISP_PS & 0.584 & 0.620 & 0.621 & 0.584 & 0.620 & 0.621 \\
\hline ANS & 0.573 & 0.525 & 0.506 & 0.573 & 0.525 & 0.506 \\
\hline ANS_PS & 0.584 & 0.620 & 0.621 & 0.584 & 0.620 & 0.621 \\
\hline ANS_mC & 0.573 & 0.525 & 0.506 & 0.573 & 0.525 & 0.506 \\
\hline
\end{tabular}

\# ANS_PS and ANS_PS* are identical in this problem. 


\section{CLOSURE}

In this paper, a six-node pentagonal solid-shell element is formulated. With the commonly adopted truncations applied to the strain components and the Jacobian determinant, a generalized laminate stiffness matrix can be defined. Assumed natural strain method is employed to alleviate shear and trapezoidal lockings of the element. A modified generalized laminate stiffness matrix is derived that resolves not only the thickness locking but also some abnormalities in laminated material analyses. Popular benchmark tests for plate/shell elements modelled by cross-diagonal meshes are exercised. The proposed element is close in accuracy to other state-of-the-art three-node degenerated shell elements.

The robustness with respect to shear locking of the present solid-shell element and other threenode degenerated shell elements depends strongly on the adaptation of the cross-diagonal mesh. Fortunately, this limitation is least subtle for meshes containing mixed element types as the eightnode elements should be dominating.

Acknowledgment - The financial support of the Research Grant Council of Hong Kong in the form of an Earmarked Grant (project no. HKU 7111/99E) is gratefully acknowledged. 


\section{REFERENCES}

1. M.F.Ausserer, S.W.Lee, An eighteen-node solid element for thin shell analysis, Inter. J. Numer.Methods Engrg, 26 (1988) 1345-1364.

2. K.Y.Sze, A.Ghali, An hexahedral element for plates, shells and beams by selective scaling, Inter.J.Numer.Methods Engrg, 36 (1993) 1519-1540.

3. H.C.Park, C.Cho, S.W.Lee, An efficient assumed strain element model with six dof per node for geometrically nonlinear shells, Inter.J.Numer.Methods Engrg. 38 (1995) 4101-4122.

4. D.Roehl, E.Ramm, Large elasto-plastic finite element analysis of solids and shells with the enhanced assumed strain concept, Inter.J.Solids \& Structures. 33 (1996) 3215-3237.

5. P.Betsch, F.Gruttmann, E.Stein. A 4-node finite shell element for the implementation of general hyperelastic 3D-elasticity at finite strains, Comput.Methods Appl.Mech.Engrg. 130 (1996) 5779.

6. K.Y.Sze, S.Yi, M.H.Tay, An explicit hybrid-stabilized eighteen-node solid element for thin shell analysis, Inter.J.Numer.Methods Methods Engrg., 40 (1997) 1839-1856.

7. R.Hauptmann, K.Schweizerhof, A systematic development of solid-shell element formulations for linear and non-linear analysis employing only displacement degrees of freedom, Inter.J. Numer.Methods Engrg., 42 (1988) 49-69.

8. K.Y.Sze, L.Q.Yao, S.Yi, A hybrid-stress ANS solid-shell element and its generalization to smart structure modeling, Inter.J.Numer.Methods Engrg., 48 (2000) 545-582.

9. K.Y.Sze, L.Q.Yao, S.N.Atluri, Development of solid-shell elements with particular reference to thickness strain, Computational Mechanics, accepted for publication.

10. R.H.MacNeal, A theorem regarding the locking of tapered four-noded membrane elements, Inter.J.Numer.Methods Engrg., 24 (1987) 1793-1799.

11. K.Y.Sze, On immunizing five-beta hybrid stress elements from 'trapezoidal locking' in practical analyses, Inter.J.Numerical Methods Engrg., 47 (2000) 907-920.

12. T.H.H.Pian, Finite elements based on consistently assumed stresses and displacements, Finite Elements in Analysis \& Design, 1 (1985) 131-140.

13. S.H.Lo, C.K.Lee, On using meshes of mixed element types in adaptive finite element analysis, Finite Elements in Analysis \& Design, 11 (1992) 307-336.

14. J.P.Coyette, Private Communication to K.Y.Sze, September 1999.

15. T.J.R.Hughes, R.L.Taylor, The linear triangular bending element, in J.R.Whiteman (ed.) Proc. $4^{\text {th }}$ MAFELAP Conf., (1982) 127-142.

16. A.F.Saleeb, T.Y.Chang, S.Yingyeunyong, A mixed formulation of $\mathrm{C}^{0}$-linear triangular plate/ shell element - the role of edge shear constraints, Inter.J.Numer.Methods Engrg., 26 (1988) 1101-1128.

17. M.L.Liu, C.W.S.To, Hybrid strain based three-node flat triangular shell elements, Finite Elements in Analysis \& Design, 17 (1994) 169-203.

18. K.J.Bathe, E.N.Dvorkin. A formulation of general shell elements - the use of mixed interpolation of tensorial components. Inter.J.Numer.Methods Engrg., 22 (1986) 697-722.

19. K.Y.Sze, D.Zhu, A quadratic assumed natural strain curved triangular shell element, Computer Methods in Applied Mechanics \& Engineering, 174 (1999) 57-71.

20. R.H.MacNeal, R.L.Harder, A proposed standard set of problems to test finite element accuracy. 
Finite Elements in Analysis \& Design, 1 (1985) 3-20.

21. S.P.Timoshenko, S.Woinowsky-Krieger, Theory of Plates and Shells, 2nd Edn., McGraw-Hill, New York, 1970.

22. S.P.Timoshenko, J.N.Goodier, Theory of Elasticity, 3rd Edn., McGraw-Hill, New York, 1982 


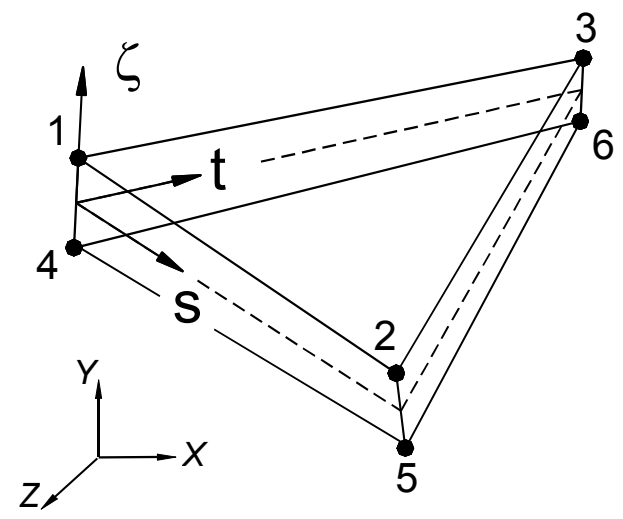

Figure 1: Six-node solid-shell element.

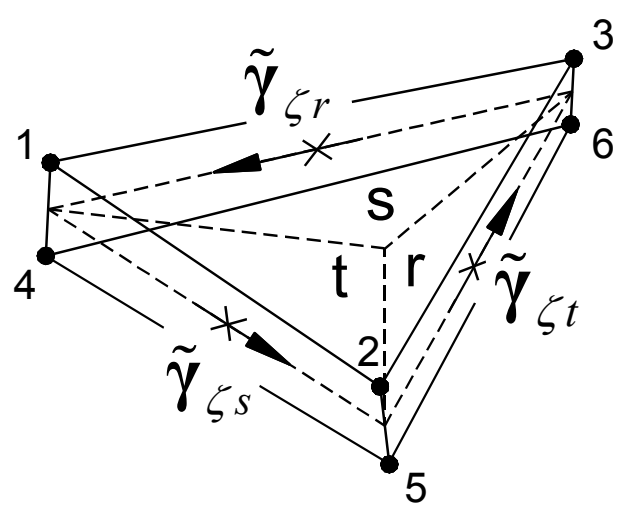

Figure 2: The sampled natural transverse shear strains.

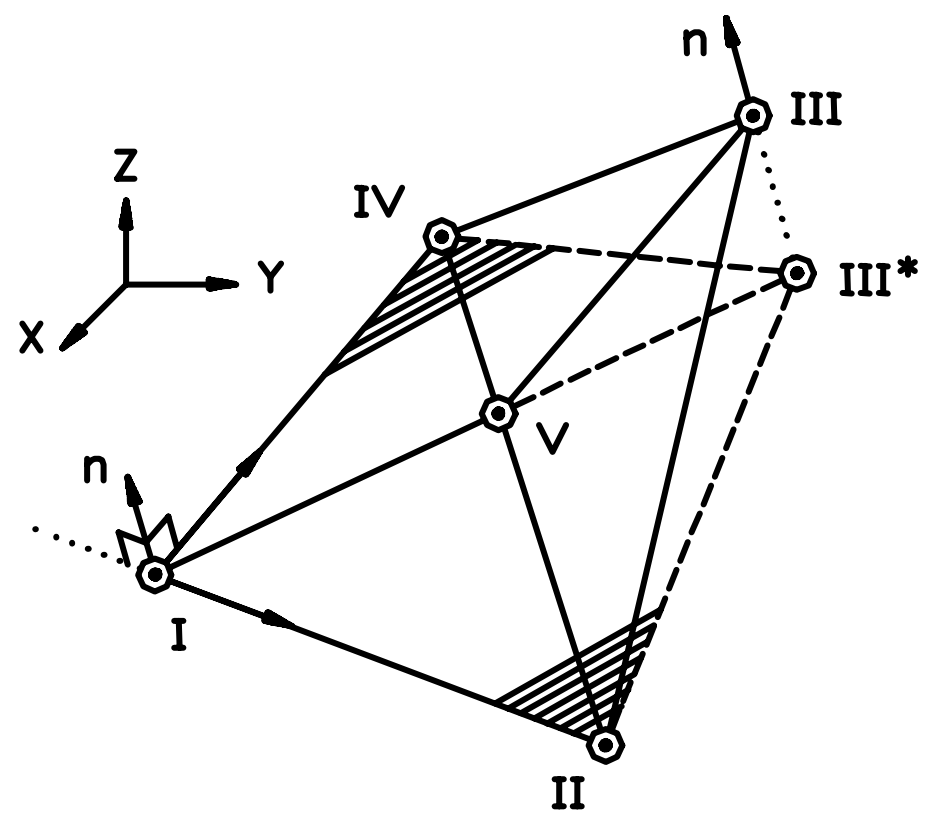

I, II, III*, IV

and $V$ are

coplanar.

I, V and III

are collinear.

II, V and IV

are collinear.

Figure 3: Location of the interior node for a quadrilateral defined by nodes I, II, III and IV. 


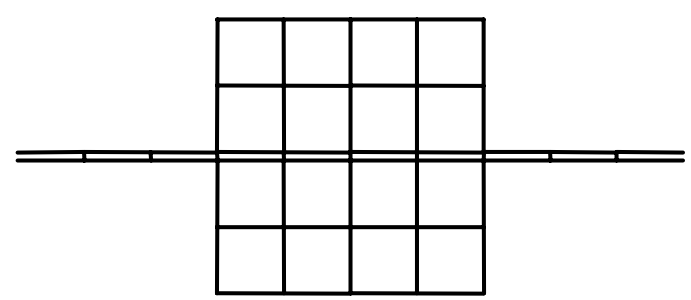

Figure 4: Solid-shell elements sandwiched by solid elements.

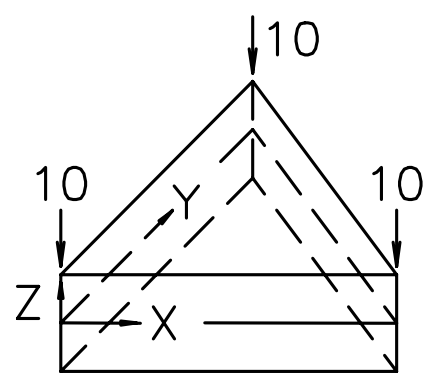

Figure 5: A right-angle triangular prism subjected to compressive loading.
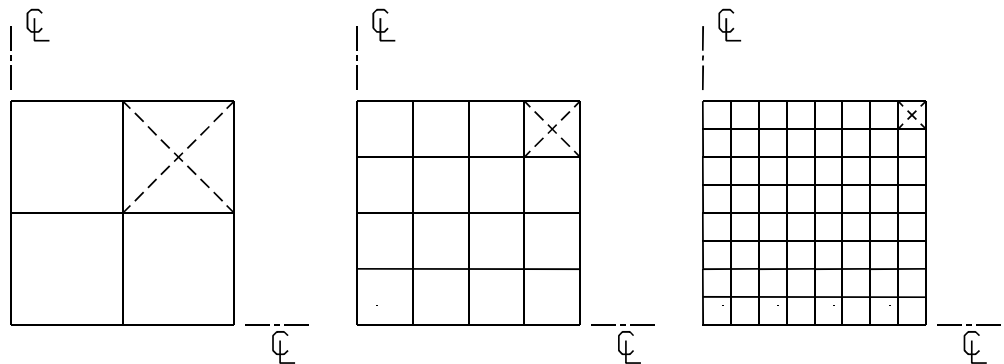

$2 \times 2$

$4 \times 4$

$8 \times 8$

Figure 6: Meshes for modelling a quadrant of a square plate.

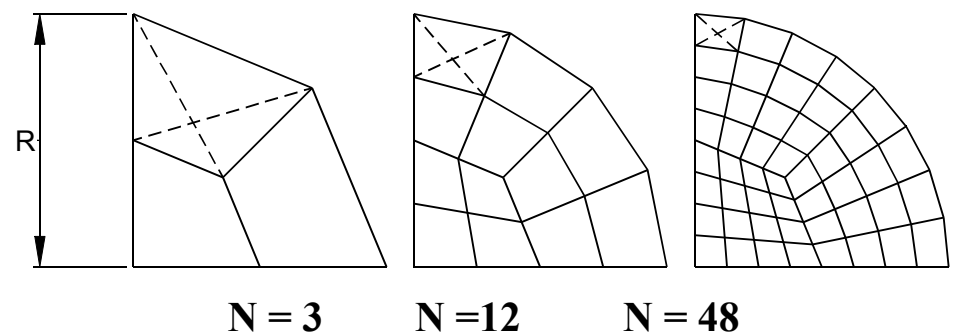

Figure 7: Meshes for modelling a quadrant of a circular plate. 


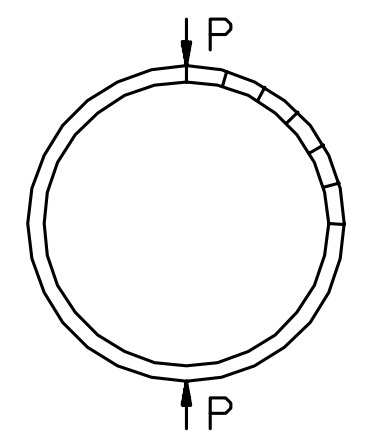

Figure 8: A unit width laminated ring-shell pinched by line forces.
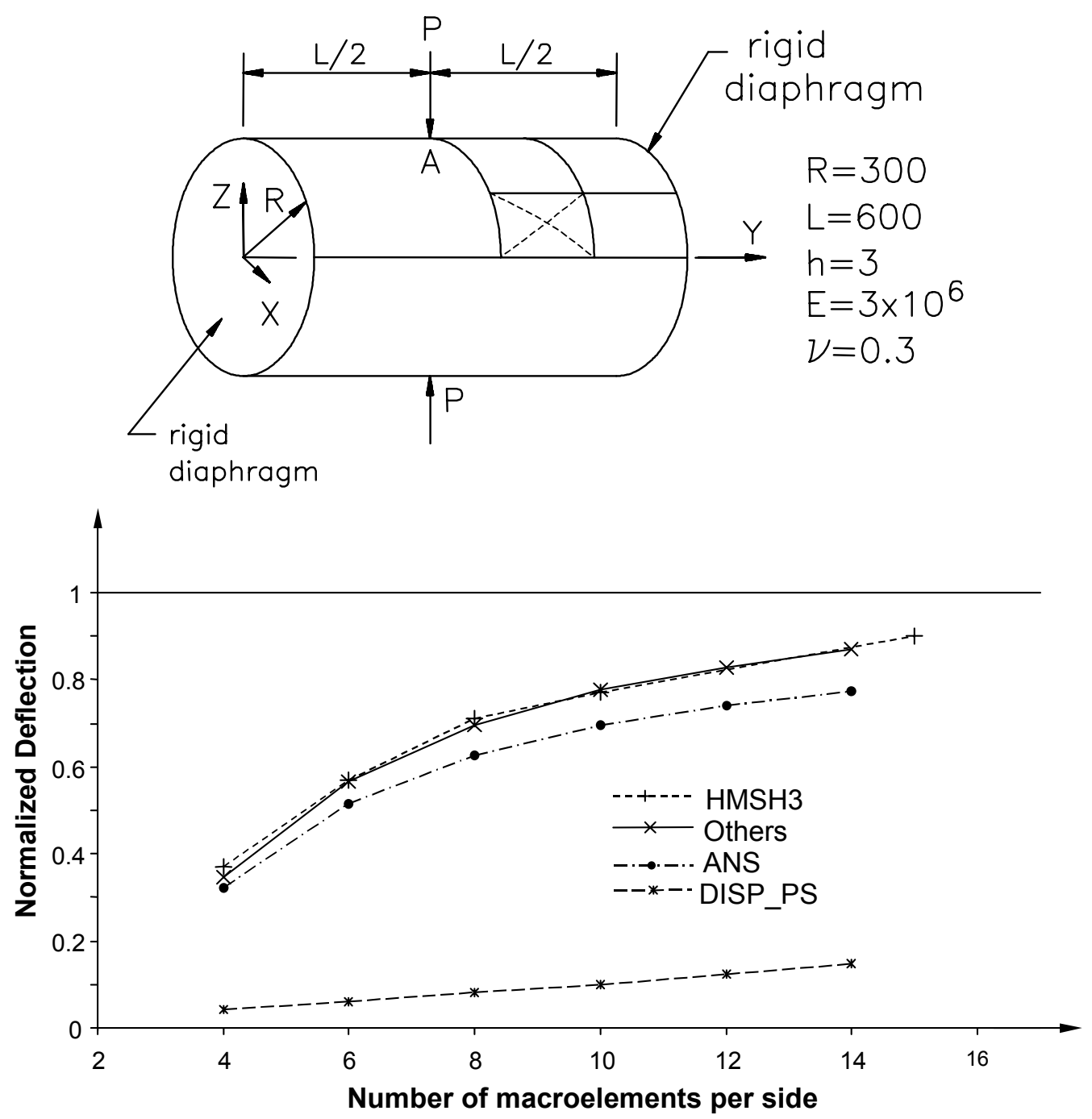

Figure 9: Normalized deflections of the pinched cylinder at point $A$. 


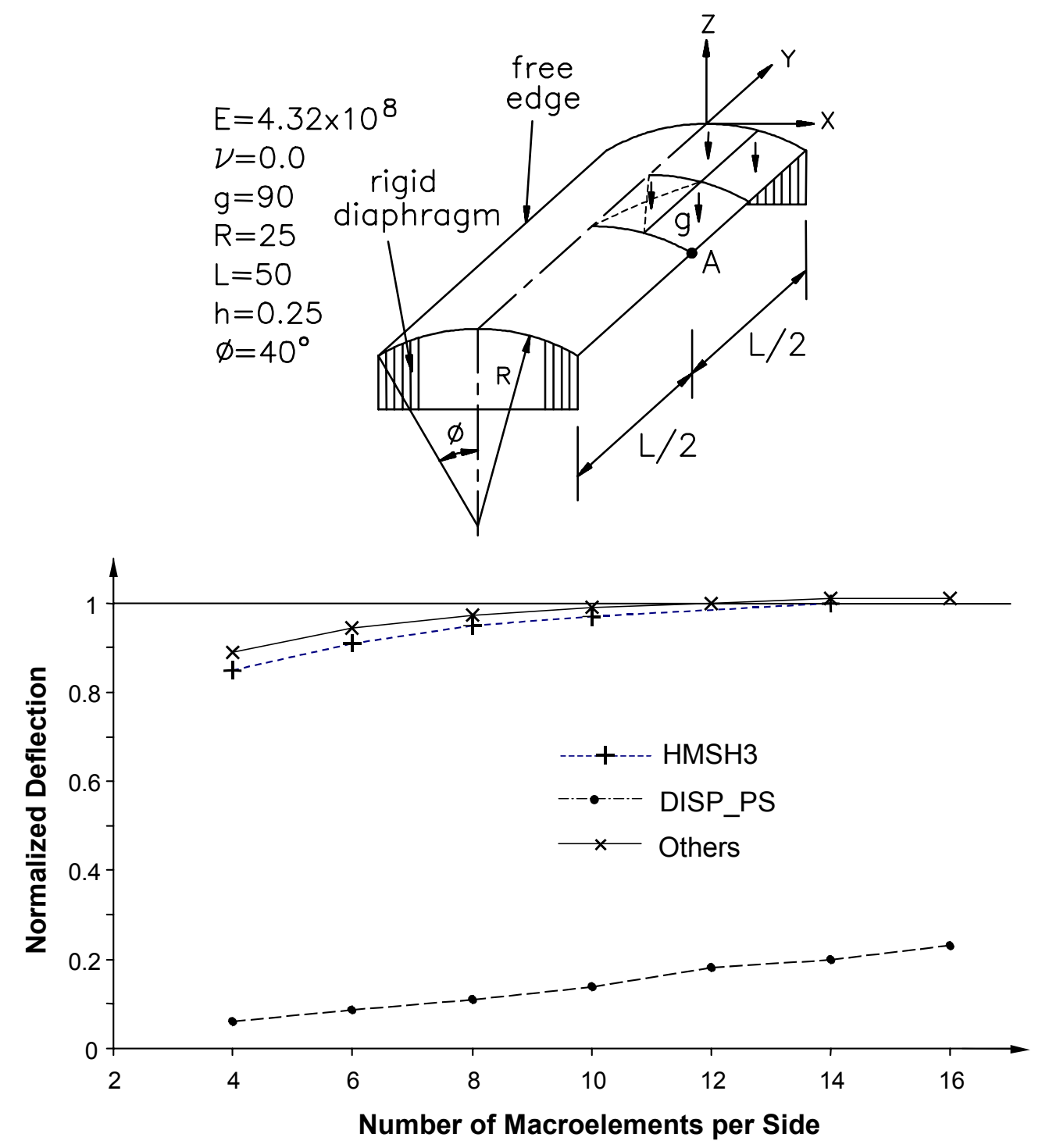

Figure 10: Normalized deflections of Scordelis-Lo cylindrical roof at point A. 

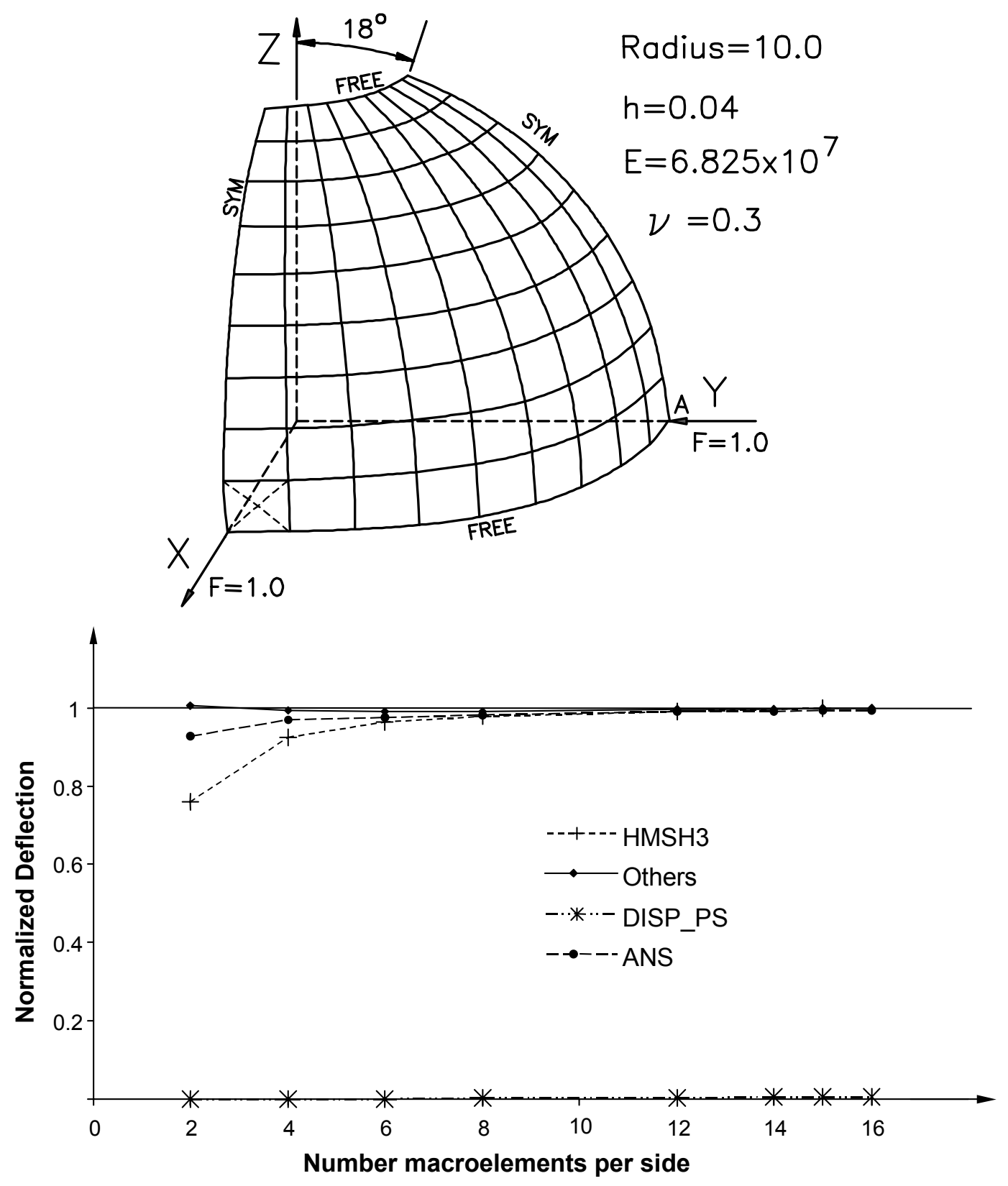

Figure 11: Normalized deflections of the hemispherical shell at point A. 


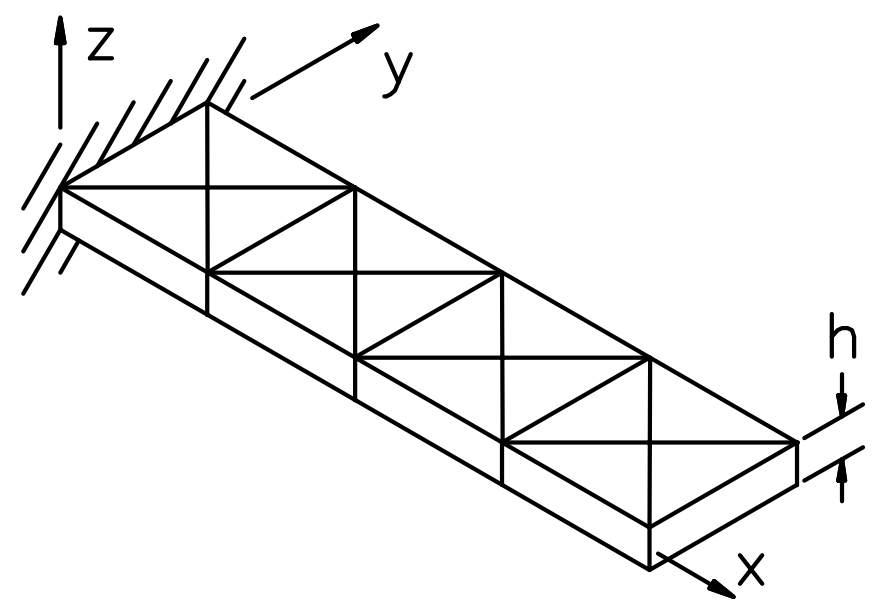

Figure 12: A $4 \times 1 \times$ h cantilever panel modelled by four macroelements. 